CLINICAL REPORT

\author{
J.L. Jaremko \\ L.B.O. Jans \\ L.T. Coleman \\ M.R. Ditchfield
}

\section{Value and Limitations of Diffusion-Weighted Imaging in Grading and Diagnosis of Pediatric Posterior Fossa Tumors}

\begin{abstract}
SUMMARY: DWI reportedly accurately differentiates pediatric posterior fossa tumors, but anecdotal experience suggests limitations. In 3 years, medulloblastoma and JPA were differentiated by DWI alone in $23 / 26$ cases ( $88 \%)$. Ependymoma $(n=5)$ could not be reliably differentiated from medulloblastoma or JPA. A trend toward increased diffusion restriction in higher grade tumors (1/14 grade I, $7 \% ; 9 / 12$ grade IV, $75 \%$ ) had too much overlap to predict the grade of individual cases. The overlap in ADC between tumor types appeared partly due to technical factors (in small, heterogeneous, calcific, or hemorrhagic tumors) but also likely reflected true histologic variability, given that our 3 overlap cases included a desmoplastic medulloblastoma, an anaplastic ependymoma, and a JPA with restricted diffusion in its nodule. Simple structural features (macrocystic tumor, location off midline) aided in distinguishing JPA from the other tumors in these cases.
\end{abstract}

ABBREVIATIONS: $A D C=$ apparent diffusion coefficient; $A D C$ mean $=$ mean value of $A D C ; A D C-$
min = minimum value of $A D C ; D W I=$ diffusion-weighted imaging; FLAIR = fluid-attenuated
inversion recovery; JPA = juvenile pilocytic astrocytoma; $W H O=$ World Health Organization
D WI might, in theory, effectively distinguish tumor types and histologic grades because higher grade tumors with more densely packed cells should have increasingly restricted diffusion (with a lower ADC). ${ }^{1}$ Unfortunately, it is wellknown that overlap between tumor grades and types is generally too great to specifically diagnose individual brain tumors with DWI alone, in adults ${ }^{2-5}$ or children..$^{6-8}$ In contrast, there are studies suggesting that DWI may be highly accurate in tumor diagnosis in the pediatric posterior fossa. One recent study showed no overlap between ADC values in the 3 main pediatric posterior fossa tumors: medulloblastoma $(n=8)$, JPA $(n=17)$, and ependymoma $(n=5) .{ }^{9}$ Two smaller studies had similar results; 1 had $n=12^{10}$ and 1 found no overlap between ADC values in ependymoma $(n=6)$ and primitive neuroectodermal tumor $(n=9$, including medulloblastoma). ${ }^{3}$

Our anecdotal experience, from several years of routine DWI in pediatric brain tumors, has been that ADC values have not been as helpful as in these reports. We performed a retrospective study to clarify the reasons for this discrepancy, first optimizing our ADC measurement technique to minimize overlap, then individually examining overlap cases to find contributing factors. We hypothesized that overlap cases would be infrequent and would have obvious technical or histologic causes and that anatomic tumor features could supplement DWI to provide accurate diagnosis in these cases.

Received January 18, 2010; accepted after revision April 1.

From the Department of Medical Imaging (J.L.J., L.T.C.) and Murdoch Children's Research Institute (M.R.D.), Royal Children's Hospital, Parkville, Victoria, Australia; Department of Radiology and Medical Imaging (L.B.O.J.), Ghent University Hospital, Gent, Belgium; and Department of Diagnostic Imaging (L.T.C.), Monash Medical Centre, Clayton, Victoria, Australia.

Please address correspondence to Jacob L. Jaremko, MD, Medical Imaging, Royal Children's Hospital, 2nd Floor, Main Block Flemington Rd, Parkville, Vic, Australia 3031; e-mail: jjaremko@rch.org.au

DOI 10.3174/ajnr.A2155

\section{Case Series}

The study was approved by the hospital Human Research Ethics Committee. At a large tertiary referral pediatric hospital, we examined initial pre-resection imaging and pathology reports for all children who had surgical biopsy or resection of a tumor located in the cerebellum and/or fourth ventricle from 2006 to 2009. Patient ages at the time of initial MR imaging ranged from 1 month to 15.6 years (mean, 5.8 years). There were 17 girls and 23 boys. Patients with JPA ( $8.2 \pm 4.2$ years) were significantly older than those with ependymoma (3.6 \pm 1.6 years, $P<.01)$ or medulloblastoma $(4.8 \pm 3.1$ years, $P<$ $.05)$. Only 1 in 5 patients with ependymoma was female (20\%), compared with nearly equal sex ratios in JPA (50\%) and medulloblastoma (44\%), a difference that was not statistically significant.

All patients were imaged on $1.5 \mathrm{~T}$ or $3 \mathrm{~T}$ magnets and had spin-echo T1- and T2-weighted and postgadolinium T1weighted images available. All except 4 patients also had FLAIR images. All patients had DWI performed with an echoplanar-base sequence by using gradient fields of $b=0,500$, and $1000 \mathrm{~s} / \mathrm{mm}^{2}$. Most had these 3 sets of trace images and an ADC map available. To be included in at least part of the analysis, patients were required to have at minimum the initial trace b1000 images. Data from spectroscopy (10 patients) and CT (14 patients) were not assessed. As a referral center, we often receive images from other institutions loaded digitally into our computer archive system in standard format. These were judged adequate for analysis if sequences were appropriate and of similar visual quality to images from our own hospital.

Images were assessed by a pediatric radiologist (J.L.J.) blinded to the pathologic diagnosis after initial planning with a senior pediatric radiologist (L.B.O.J.). Several lesion characteristics were assessed by using T1, T2, postgadolinium T1, and FLAIR (if available) images. We noted whether the tumor was centered at midline or laterally and whether it involved cerebellopontine angles. The cystic component was graded as none (entirely solid), macrocystic (single dominant cyst), or 
microcystic/multicystic. Enhancement was graded as present ( $>25 \%$ of the tumor), minimal (only a few enhancing foci, $<25 \%$ of the tumor), absent, or peripheral (ring enhancement only). Peritumoral edema was graded as none, mild, or extensive. T2 signal intensity of the tumor was graded qualitatively as hyperintense, isointense, or hypointense with respect to uninvolved gray matter. We noted a tumor as likely to be hemorrhagic if it contained areas of T1 signal intensity substantially higher than that of surrounding tissues on pregadolinium sequences $^{3,11}$ and as likely to be necrotic if there was noncystic nonenhancing tissue centrally within a predominantly enhancing area of tumor. ${ }^{3}$ We wanted to record whether a tumor was calcific, but the signal intensity associated with calcification is variable and nonspecific. ${ }^{12}$ Accordingly, we recorded whether the tumor contained areas of very low T1 and T2 signal intensity, which would indicate attenuated calcification or blood products from old hemorrhage.

Diffusion imaging analysis was performed by using Synapse, Version 3.2, 2009 (Fujifilm Medical Systems, Stamford, Connecticut). We sought the area of greatest diffusion restriction (lowest ADC) within the solid tumor component and avoided peritumoral edema. First, we recorded whether restricted diffusion was visually obvious within the tumor, defined as an area of bright signal intensity on $b=1000$ images and a corresponding low ADC (a dark area on the ADC map). For numeric ADC analysis, we used the following approach based on our literature review: 1) Examine T1, T2, and postgadolinium sequences to identify and avoid areas likely to be cystic, hemorrhagic, calcific, necrotic, or peritumoral edema. 2) Find the section with the lowest ADC visually within areas of tumor not excluded in step 1.3) Create a mildly elliptic region of interest $40-45 \mathrm{~mm}^{2}$ in this section, long axis less than twice the short axis length, and record the ADC value in this region of interest. 4) If the low ADC area is large, move the region of interest slightly within it and record up to 3 sample ADC values. 5) Repeat steps 3 and 4 for the area of highest ADC in the tumor. 6) Repeat steps 3 and 4 within an area of distinct intermediate ADC if any. This gives up to 9 ADC values within each tumor. From this, we computed the ADCmin across all regions. We also computed the ADCmean within the region of lowest ADC (from up to 3 samples of this area obtained in the first iteration of step 4). Note was made of any difficulties in selecting the region of interest, such as artifacts or small lesion size. One pediatric radiologist (J.L.J.) measured regions of interest for all patients, and another (L.B.O.J.) measured regions of interest for a randomly selected subset of 20 patients, by using the same rules.

On the basis of the original pathology report, tumor diagnosis and the associated WHO grade were recorded for each patient per recent guidelines ${ }^{13}$ : Ependymomas are grade 2 unless anaplastic, which are grade 3; medulloblastomas are grade 4; and astrocytoma grade varies by histologic findings. Any cases with inconclusive results were discussed with the pathologist to clarify diagnosis.

Statistical analysis used Excel 2007 (Microsoft, Redmond, Washington) and the Statistical Package for the Social Sciences, Version 9 (SPSS, Chicago, Illinois). Basic descriptive statistics were computed, with results reported as mean $\pm \mathrm{SD}$. Differences between means of continuous variables, such as between ADC values in 2 tumor types, were tested for significance by the nonparametric Wilcoxon Mann-Whitney test (rather than the Student $t$ test, to avoid assumptions regarding normal distribution of data) at $P=.05,1$-tailed. Given the small sample sizes, the difference between proportions by using binary variables (eg, frequency of restricted diffusion in each tumor type) was tested by using the Fisher exact test rather than the $\chi^{2}$ test. To test interobserver variability in the minimum ADC values, we calculated the $\kappa$ statistic.

Of the 40 tumors meeting inclusion criteria, we had 17 JPAs, 10 medulloblastomas, and 8 ependymomas. The other 5 tumors included atypical teratoid rhabdoid tumors $(n=2)$, ganglioglioma $(n=1)$, grade 2 glioma without pilocytic features $(n=1)$, and a glioblastoma multiforme $(n=1)$. Most patients had no prior brain surgery at the time of MR imaging, but 7 patients had undergone shunt placement (typically in a lateral ventricle far from the tumor). One patient with a combined cerebellar/brain stem pilocytic astrocytoma had a previous partial debulking surgery, but on review of the images, the large residual tumor, away from the resection site, was judged to be acceptable for inclusion in the study. This data point was not an outlier in subsequent analysis. Thirty-four of $40(85 \%)$ tumors had DWI performed preoperatively. One was uninterpretable due to artifacts from braces, and 1 patient had $b=1000$ images saved without the ADC map. This left $32 / 40$ (80\%) interpretable ADC maps. Subjective analysis was still possible on the patient with only the $b=1000$ images saved, with the caveat that bright signal intensity on $b=1000$ could still represent $\mathrm{T} 2$ shinethrough artifacts rather than true restricted diffusion.

All of the JPAs, ependymomas, and medulloblastomas were T2 hyperintense, and most $(29 / 35,83 \%)$ had a cystic component. Most also had strong enhancement throughout their solid component, though 1 medulloblastoma, 1 ependymoma, and 3 JPAs had only traces of enhancement and 1 JPA had only rim enhancement. Only 5/35 tumors (14\%) had areas suspicious for calcification or hemorrhage. All medulloblastomas and all but 5 tumors of other types had peritumoral edema. These features were nondifferentiating. However, a tumor centered off midline was more likely a JPA (midline location JPA $=47 \%$, others $=80 \%-89 \% ; P<.05$ for JPA versus medulloblastoma). Ependymomas and JPAs were more likely to involve the cerebellopontine angles than medulloblastomas (40\% and 35\% versus $22 \%$ ), and a macrocystic component favored a JPA (53\% versus 20\%-22\%), but these trends did not reach statistical significance.

Interobserver variability between 2 pediatric radiologists (J.L.J., L.B.O.J.) in ADCmin in a subset of 20 tumors was $20 \pm$ $64 \times 10^{-6} \mathrm{~mm}^{2} / \mathrm{s}$ (range, -112 to $+166 ; 2.4 \% \pm 7.4 \%$ of the ADCmean). However, much of this variability was in 4 of the 20 tumors, 2 heterogeneous with high ADCmin and 2 with low $\mathrm{ADCmin}$. ADC measurements in the remaining tumors varied much less. When answering the question of whether ADCmin was less than a threshold value, we had perfect interobserver agreement $(\kappa=1.0)$ at threshold $\mathrm{ADCmin}=800$ and excellent agreement $(1$ disputed case $)$ at $\mathrm{ADCmin}=900(\kappa=$ 0.90 ). Time to measure ADC ranged from seconds to $5 \mathrm{~min}$ utes per scan, decreasing with increased observer experience.

Diffusion restriction was highly correlated to WHO tumor grade but with overlap between grades. Visual and quantita- 


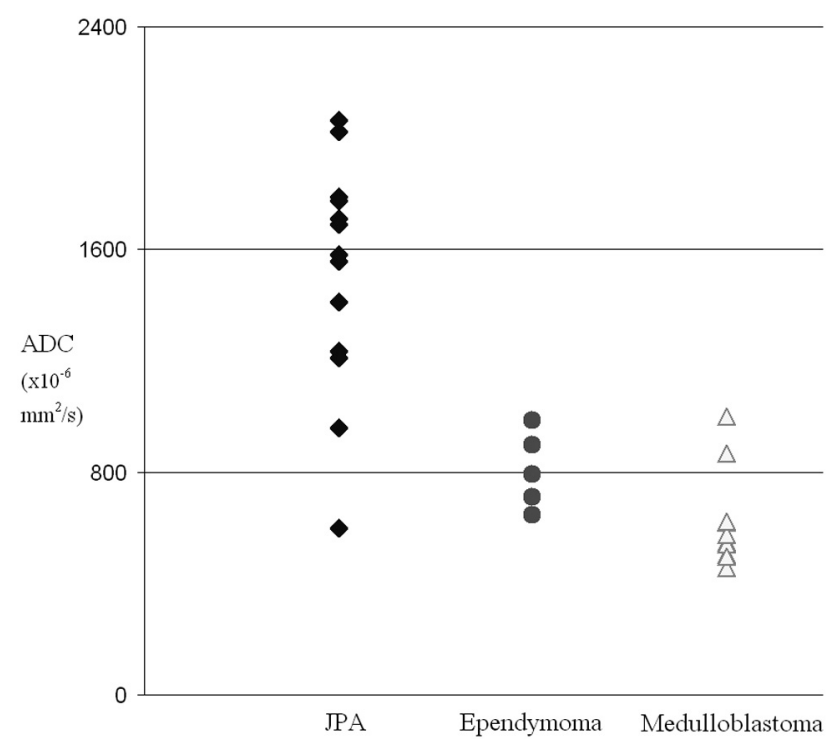

Fig 1. Relation of diffusion restriction to tumor grade in pediatric posterior fossa tumors. The presence of diffusion restriction is identical at visual assessment or at threshold $\mathrm{ADC}<800 \times 10^{-6} \mathrm{~mm}^{2} / \mathrm{s}$.

tive assessment of diffusion restriction produced identical results at a threshold of $800 \times 10^{-6} \mathrm{~mm}^{2} / \mathrm{s}$. Only 1 in $14(7 \%)$ of the WHO grade 1 tumors had restricted diffusion versus 9 in $12(75 \%)$ WHO grade 4 tumors. Two-thirds (4/6, 67\%) of the intermediate-grade tumors, which were ependymomas and 1 glioma, showed diffusion restriction. Diffusion was restricted in 1 of 2 classic (WHO grade 2) and 2 of 3 anaplastic (grade 3 ) ependymomas. The single patient with a $b=1000$ trace and no ADC map had a JPA with no visible diffusion restriction.

The relation between tumor type and diffusion restriction is shown in Fig 1. JPAs had significantly higher ADCmin than either ependymomas (grade 2 or $3, P<.01$ ) or medulloblastomas (grade $4, P<.0001$ ), but ADC values overlapped among the 3 tumor types. Ependymoma had a slightly but significantly $(P<.05)$ higher ADCmin than medulloblastoma, with extensive overlap. Use of ADCmean instead of ADCmin increased the amount of overlap. Diffusion restriction was seen in grade 2 ( 1 of 2 ) and 3 ( 2 of 3 ) ependymomas, and ADC values in these 2 small subsets overlapped each other.

JPA and medulloblastoma were best differentiated at a threshold ADCmin $<800 \times 10^{-6} \mathrm{~mm}^{2} / \mathrm{s}$ (Fig 1), where 1 JPA lay above this threshold and 2 medulloblastomas lay below it. These were reviewed in detail. The JPA outlier had clear diffusion restriction within the nodular component of a macrocystic tumor. The 2 outlier medulloblastomas included 1 with a desmoplastic subtype and 1 that presented with diffuse metastasis at initial scanning, in which each individual lesion was small and difficult to assess quantitatively. One other JPA overlapped with ependymoma. This tumor was heavily calcified and/or hemorrhagic, resulting in difficulty selecting an appropriate region of interest for reproducible ADC values. It had a large off-midline component in the left cerebellar peduncle.

\section{Discussion}

We confirmed that DWI is the single most useful sequence for differentiating pediatric posterior fossa tumors and that as ex- pected, diffusion restriction is rare in grade 1 tumors and common in grade 4 tumors. We confirmed others' findings ${ }^{3,9}$ that JPA and medulloblastoma are easily differentiated by ADC, but unlike those studies, we found that ADC (whether minimum or mean) did overlap. Similar overlap is also visible in data presented in a more recent study. ${ }^{8}$ Fortunately, other imaging characteristics were helpful in diagnosis. One JPA had restricted diffusion in the tumor nodule, but the correct diagnosis was suggested by structural features favoring JPA (ie, macrocystic tumor and location off-midline). Of the 2 cases of medulloblastoma that did not have clear diffusion restriction, 1 presented with multiple metastases. Because ependymoma shows no distant disease in $>90 \%$ of cases, ${ }^{14}$ this presentation favors medulloblastoma. The other was a desmoplastic medulloblastoma, a histologically less aggressive subtype with better prognosis than the classic type. ${ }^{15}$ The single desmoplastic medulloblastoma in the series of Rumboldt et $\mathrm{al}^{9}$ did have restricted diffusion. Whether there are differences in imaging features of desmoplastic and conventional medulloblastoma is not well studied, but one might expect the less aggressive desmoplastic type to have less highly restricted diffusion than the classic type.

Contrary to several prior reports, ${ }^{3,6,9}$ and similar to a more recent study, ${ }^{8}$ we did find overlap between the ADC of medulloblastoma and ependymoma. Within ependymomas, there was overlap between ADC values of the classic type (WHO grade 2, one-half restricted diffusion) and anaplastic type (WHO grade 3, two-thirds restricted diffusion). None of the 6 grade 2 ependymomas in a prior series had visually restricted diffusion. ${ }^{6}$ Distinguishing classic and anaplastic ependymomas is difficult for pathologists, resulting in the incidence of anaplastic ependymoma varying from $7 \%$ to $89 \%$ in reported series. ${ }^{14}$ The $60 \%$ proportion of anaplastic ependymomas in our study is similar to the $43 \%-55 \%$ reported in large series. ${ }^{16,17}$ Whether anaplastic ependymoma has poorer prognosis than classic ependymoma is also controversial. ${ }^{14}$ Given the wide histologic and prognostic spectrum of ependymoma, diffusion characteristics of ependymoma also have a wide range overlapping other tumor types, as seen in this study. In contrast to Kan et al, ${ }^{6}$ who suggested that DWI should be routinely performed as a means of distinguishing ependymomas and medulloblastomas, we found no features that would reliably make this distinction.

That some groups have found DWI more useful than others is likely partly due to differences in the ADC measurement technique. Ideally, for routine clinical use this should be rapid and should not require a specialized postprocessing workstation. The simplest approach ${ }^{6}$ is to record whether restricted diffusion is visually present (bright area on the $b=1000$ trace image and a corresponding dark area on ADC map). This can be done even for older or outside studies in which only filmed or scanned images are available. In our data, this visual technique identified diffusion restriction with results identical to those of quantitative ADC measurement at the threshold ADCmin $=800 \times 10^{-6} \mathrm{~mm}^{2} / \mathrm{s}$. If ADC is quantified numerically, care must be taken in selecting the appropriate region of interest. Cystic, necrotic, or hemorrhagic areas must be avoided. ${ }^{3,5,8,18}$ Most groups use a larger region than a single voxel, to reduce the effect of noise or misregistration artifacts. ${ }^{5}$ A large series on medulloblastoma, ${ }^{11}$ which did use single-voxel anal- 
ysis, had much lower ADC $(\mathrm{ADCmean}=470 \pm 160)$ than that in other reports. A 5-voxel region of interest ${ }^{2}$ is still small $(\sim 14$ $\mathrm{mm}^{2}$ ). We followed others who used a region of interest of 40-60 $\mathrm{mm}^{2}$ per 4 - to 8 -mm section. ${ }^{4,5}$ The most valuable measurement has been the minimum ADC value in the tumor, ${ }^{4,5}$ while tumor heterogeneity can be characterized by the maximal ADC value and range of ADC values within the tumor. ${ }^{5}$ ADCmean within the tumor, such as from randomly selected areas within the tumor, ${ }^{3}$ is an alternative to ADCmin. Overall, region-of-interest selection and the details of ADC calculation are likely to have an important influence on tumor analysis.

Our technique sought the true minimum ADC by numerically exploring the region of greatest restriction visually within areas of solid tumor, which we expect to be the most physiologically meaningful measurement. As we hoped, this resulted in lower $\mathrm{ADC}$ values and a narrower range of $\mathrm{ADC}$ variation than most other reports. Our optimal threshold for distinguishing medulloblastoma and JPA, $\mathrm{ADCmin}=800 \times$ $10^{-6} \mathrm{~mm}^{2} / \mathrm{s}$, was lower than the threshold of 900 used by Rumboldt et al, ${ }^{9}$ likely because they used ADCmean rather than ADCmin. The threshold separating primitive neuroectodermal tumors and ependymoma in a study mixing pediatric and adult patients was also higher, at ADCmin $=1000$, when the authors used a single region of interest placed by consensus. ${ }^{3}$ Our ADCmeans for specific tumors were notably lower than those in other groups for ependymoma. In a recent study that used a single $43 \mathrm{~mm}^{2}$ region of interest, ependymoma ADC ranged from $\sim 750$ to $\sim 1300$, overlapping JPA and medulloblastoma. ${ }^{8}$ Our targeted search for ADCmin in these heterogeneous tumors resulted in a tighter range of $\mathrm{ADC}$ for ependymoma (ADCmin $=650-990)$, but this still overlapped the other tumor types.

The interobserver variability we noted in ADC measurements was substantial at the extremes (tumors with highly restricted diffusion or marked heterogeneity), but despite this, there was excellent interobserver agreement on whether the threshold for restricted diffusion was met $(\kappa=0.9$ to 1.0$)$. Interobserver range of variation in ADCmin was similar to others: $-13 \%$ to $+19 \%$ of mean ADCmin versus $-16 \%$ to $+21 \% .^{5}$ It seems that the presence of restricted diffusion can be assessed much more reliably than its precise numeric extent.

We did not observe the $75 \%$ male predominance in medulloblastoma seen in a larger series, ${ }^{11}$ and our mean age at diagnosis was slightly less than the mean age in that series but well within its age range $(6.5 \pm 4.5$ years $)$. Rumboldt et $\mathrm{al}^{9}$ had a higher mean age for all tumors but included young adult patients up to 23 years of age. These differences are unlikely to be meaningful.

Measurement of ADC is no longer an experimental or time-consuming process. We were able to measure ADC rapidly with routine image-viewing software and no postprocessing. Visual assessment of diffusion restriction produced results identical to $\mathrm{ADC}$ quantification at threshold $\mathrm{ADCmin}=$ $800 \times 10^{-6} \mathrm{~mm}^{2} / \mathrm{s}$, justifying a simple qualitative approach. ${ }^{4}$ Patients with braces, small tumors, or extensively hemor- rhagic/calcific tumors may not have interpretable ADC, but $90 \%$ of patients in our study were assessable.

In conclusion, this study showed that contrary to some recent reports, even with technique optimized to find the objective minimum ADC in each tumor, there was true overlap between diffusion characteristics of JPA, ependymoma, and medulloblastoma. Overlap could not be accounted for solely by technical difficulties in ADC measurement (small, hemorrhagic, or calcific tumors) but included variation in tumor pathology (desmoplastic medulloblastoma, anaplastic ependymoma, and restricted diffusion in a JPA tumor nodule). DWI remained highly useful in the diagnosis of pediatric posterior fossa tumors, accurately distinguishing JPA from the other 2 main tumor types when combined with simple structural features (tumor location, macrocystic morphology, metastasis), without need for advanced spectroscopic analysis.

\section{References}

1. Provenzale JM, Mukundan S, Barboriak DP. Diffusion-weighted and perfusion MR imaging for brain tumor characterization and assessment of treatment response. Radiology 2006;239:632-49

2. Server A, Kulle B, Maehlen J, et al. Quantitative apparent diffusion coefficients in the characterization of brain tumors and associated peritumoral edema. Acta Radiol 2009;50:682-89

3. Yamasaki F, Kurisu K, Satoh K, et al. Apparent diffusion coefficient of human brain tumors at MR imaging. Radiology 2005;235:985-91

4. Lee EJ, Lee SK, Agid R, et al. Preoperative grading of presumptive low-grade astrocytomas on MR imaging: diagnostic value of minimum apparent diffusion coefficient. AJNR Am J Neuroradiol 2008;29:1872-77

5. Murakami R, Hirai T, Sugahara T, et al. Grading astrocytic tumors by using apparent diffusion coefficient parameters: superiority of a one- versus twoparameter pilot method. Radiology 2009;251:838-45

6. Kan P, Liu JK, Hedlund G, et al. The role of diffusion-weighted magnetic resonance imaging in pediatric brain tumors. Childs Nerv Syst 2006;22:1435-39

7. Tzika AA, Zarifi MK, Goumnerova L, et al. Neuroimaging in pediatric brain tumors: Gd-DTPA-enhanced, hemodynamic, and diffusion MR imaging compared with MR spectroscopic imaging. AJNR Am J Neuroradio 2002;23:322-33

8. Schneider JF, Confort-Gouny S, Viola A, et al. Multiparametric differentiation of posterior fossa tumors in children using diffusion-weighted imaging and short echo-time 1H-MR spectroscopy. J Magn Reson Imaging 2007;26:1390-98

9. Rumboldt Z, Camacho DL, Lake D, et al. Apparent diffusion coefficients for differentiation of cerebellar tumors in children. AJNR Am J Neuroradiol 2006;27:1362-69

10. Gauvain KM, McKinstry RC, Mukherjee P, et al. Evaluating pediatric brain tumor cellularity with diffusion-tensor imaging. AJR Am J Roentgenol 2001;177:449-54

11. Koral K, Gargan L, Bowers DC, et al. Imaging characteristics of atypical teratoid-rhabdoid tumor in children compared with medulloblastoma. AJR Am J Roentgenol 2008;190:809-14

12. Tsuchiya K, Makita K, Furui S, et al. MRI appearances of calcified regions within intracranial tumors. Neuroradiology 1993;35:341-44

13. Rousseau A, Mokhtari K, Duyckaerts C. The 2007 WHO classification of tumors of the central nervous system: what has changed? Curr Opin Neurol 2008;21:720-27

14. Bouffet E, Perilongo G, Canete A, et al. Intracranial ependymomas in children a critical review of prognostic factors and a plea for cooperation. Med Pediatr Oncol 1998;30:319-29, discussion 329-31

15. McManamy CS, Pears J, Weston CL, et al. Nodule formation and desmoplasia in medulloblastomas-defining the nodular/desmoplastic variant and its biological behavior. Brain Pathol 2007;17:151-64

16. Ho DM, Hsu CY, Wong TT, et al. A clinicopathologic study of 81 patients with ependymomas and proposal of diagnostic criteria for anaplastic ependymoma. J Neurooncol 2001;54:77-85

17. Agaoglu FY, Ayan I, Dizdar Y, et al. Ependymal tumors in childhood. Pediatr Blood Cancer 2005;45:298-303

18. Jost SC, Ackerman JW, Garbow JR, et al. Diffusion-weighted and dynamic contrast-enhanced imaging as markers of clinical behavior in children with optic pathway glioma. Pediatr Radiol 2008;38:1293-99 